\title{
Mobility dependence on carrier density in a dilute GaAs electron gas in an in-plane magnetic field
}

\author{
Ming-Gu Lin ${ }^{\mathrm{a}}$, Chao-Ping Huanga , C.-T. Liang, ${ }^{\mathrm{a}, *}$, C.G. Smith ${ }^{\mathrm{b}}$, \\ M.Y.Simmons ${ }^{\mathrm{c}}$, D.A. Ritchie ${ }^{\mathrm{b}}$ \\ a Department of Physics, National Taiwan University, Taipei 106, Taiwan \\ ${ }^{\mathrm{b}}$ Cavendish Laboratory, Madingley Road, Cambridge CB3 OHE, UK \\ ${ }^{\mathrm{c}}$ School of Physics, University of New South Wales, Sydney 2052, Australia
}

\begin{abstract}
We report low-temperature magnetoresistivity measurements of a high-quality gated two-dimensional electron gas (2DEG). In the dilute electron density limit, we show evidence for spin polarisation in an in-plane magnetic field. Using a simple model, we estimate the Landé $g$-factor in this dilute 2DEG to be about 3.32. This enhanced Landé $g$-factor compared with that of a bulk GaAs 2D electron system (0.44) is ascribed to electron-electron interaction effects at ultra-low electron densities and the fact that over the whole measurement range $r_{\mathrm{s}}$ does not vary significantly. Moreover, we report the mobility $\mu$ dependence on electron density $n$ of a dilute electron gas at different in-plane magnetic fields. It is found that the exponent $\alpha$ in the relation $\mu \propto n^{\alpha}$ increases with increasing in-plane magnetic field. We ascribe this effect to the combination of increasing scattering and spin polarisation with increasing parallel magnetic field.
\end{abstract}

(c) 2003 Elsevier B.V. All rights reserved.

PACS: 74.40.Xy; 71.63.Hk

Keywords: Magnetoresistivity; GaAs electron gas; Landé $g$-factor

In a two-dimensional electron gas (2DEG) [1], applying an in-plane magnetic field $B_{\|}$parallel to the plane of the electron system has been proved to be a powerful technique to study spin-dependent electron transport. In the first tilted magnetic field experiment on a two-dimensional (2D) Si electron gas, the $g$-factor was found to be considerably larger than the bulk electron $g$-factor [2]. Janak [3] first proposed that this enhanced $g$-factor is due to exchange interactions amongst the electrons. In the following

\footnotetext{
* Corresponding author. Fax: +886-2-23639984.

E-mail address: ctliang@phys.ntu.edu.tw (C.-T. Liang).
}

years a number of authors [4-7] studied this interesting many-body effect. With the advent of the modulation doped GaAs/AlGaAs heterojunction [8], exchange-enhanced $g$-factors were also observed in 2D GaAs electron gases [9-11].

Recently there has been much interest in transport in dilute 2D systems [12-16]. In these studies, there are strong carrier-carrier interactions within the $2 \mathrm{D}$ systems. In an in-plane magnetic field, the 2D system shows strong magnetoresistance which is believed to be a manifestation of the spin alignment of the free carriers $[15,17,18]$. The suppression of the "metallic state" with increasing in-plane magnetic field has now become important in trying to understand the 
underlying physics of the "metallic-like conductivity" in two dimensions.

It is generally accepted that it is possible to determine the dominant scattering mechanism in a $2 \mathrm{D}$ electron system at zero magnetic field from the mobility $\mu$ dependence on carrier density $n$. For example, when $\mu \propto n^{1.5}$ then remote ionised impurity scattering dominates. To date, no such study has been conducted for a dilute $2 \mathrm{D}$ electron system in an in-plane magnetic field. In this paper, we report low-temperature magnetoresistivity measurements of a dilute 2D GaAs electron gas in which carrier-carrier interactions are much weaker compared with those in a GaAs hole gas [15] and in a Si electron gas [16]. We shall show evidence for spin polarisation in an in-plane magnetic field. Using a simple model, we estimate the Landé $g$-factor in this dilute 2DEG to be about 3.32. The enhanced value of the Landé $g$-factor in this dilute limit compared with that of a bulk 2DEG (0.44) is ascribed to electron-electron interactions and the fact that over the whole measurement range $r_{\mathrm{s}}$ does not vary significantly $\left(3.7 \leqslant r_{\mathrm{s}} \leqslant 4.7\right)$. Moreover, we present the first mobility $\mu$ dependence on electron density $n$ of a dilute electron gas at different in-plane magnetic fields. It is found that $\alpha$ increases with increasing in-plane magnetic field. We ascribe this effect to the combination of increasing scattering at the GaAs/AlGaAs interface and spin polarisation with increasing parallel magnetic field.

The measurements were performed on a gated Hall bar made from $\mathrm{GaAs} / \mathrm{Al}_{0.33} \mathrm{Ga}_{0.67}$ As heterostructure. At $V_{\mathrm{g}}=0$, the carrier concentration of the $2 \mathrm{DEG}$ was $1.53 \times 10^{11} \mathrm{~cm}^{-2}$ with a mobility of $4 \times 10^{6} \mathrm{~cm}^{2} / \mathrm{V} \mathrm{s}$ after brief illumination by a red light emitting diode. The depth of the 2DEG is $300 \mathrm{~nm}$ for our device. Experiments were performed in a top-loading ${ }^{3} \mathrm{He}$ cryostat at $T=300 \mathrm{mK}$ and the four-terminal magnetoresistivity was measured with standard phase-sensitive techniques. The in-plane magnetic field $B_{\|}$is applied parallel to the source-drain current. To check for an out-of-plane magnetic field component, we measure the Hall voltage. From this we know that the sample was aligned better than $0.1^{\circ}$ using an in situ rotating insert.

Fig. 1 shows the four-terminal magnetoresistivity $\rho_{x x}$ as a function of in-plane magnetic field $B_{\|}$ at various carrier densities $n_{\mathrm{s}}$. Let us consider the uppermost curve. We see that $\rho_{x x}$ shows a $B_{\|}^{2}$

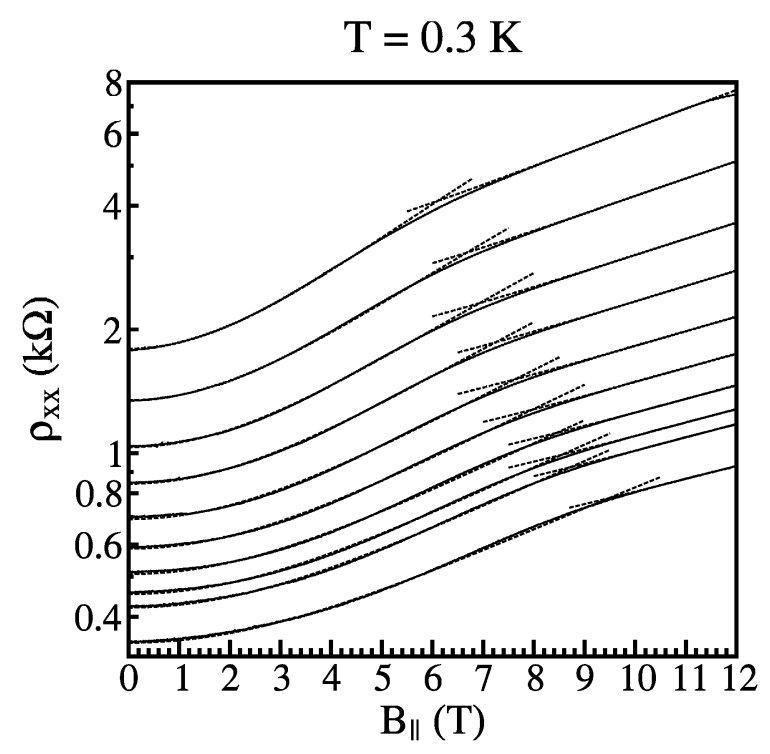

Fig. 1. $\rho_{x x}\left(B_{\|}\right)$for various carrier densities. From top to bottom: $n_{\mathrm{s}}=1.379,1.481,1.591,1.688,1.780,1.884,1.967,2.036$, 2.076 and $2.226 \times 10^{10} \mathrm{~cm}^{-2}$, respectively. Two parabolic fits for $B_{\|}<5 \mathrm{~T}$ and $>9 \mathrm{~T}$ for various $n_{\mathrm{s}}$ are shown in dotted lines.

dependence for $B_{\|}<5 \mathrm{~T}$ and shows a weaker $B_{\|}^{2}$ dependence for $B_{\|}>9 \mathrm{~T}$, as shown by the two dotted lines. It is easier to see the $B_{\|}^{2}$ dependence in both the low and high field regime when we plot $\rho_{x x}$ as a function of $B_{\|}^{2}$, as shown in Fig. 2. We ascribe the increase in $\rho_{x x}$ at low $B_{\|}$to gradual spin alignment of the 2DEG $[15,17,18]$. It is worth mentioning that in both previous work $[15,16], \rho_{x x}$ shows an exponential $B$ dependence in both low and high magnetic field regimes. We believe the fact that in our case $\rho_{x x}$ shows a $B^{2}$ dependence is due to much weaker carrier-carrier interactions compared with those in previous studies $[15,16]$. To obtain quantitative information on this spin alignment effect, we use an empirical method similar to those reported $[15,16]$, but using two parabolic fits, as shown in the two dotted lines in Fig. 2 for various $n_{\mathrm{s}}$. The interception of two parabolic fits is defined as the "crossing field" $B_{\text {cross }}$ for a certain $2 \mathrm{D}$ carrier density. We note that recently Tutuc and co-workers performed similar magneto-transport measurements on a dilute GaAs electron system in a parallel magnetic field [19]. In their system, the magnetoresistivity shows an $\exp \left(B_{\|}^{2}\right)$ dependence at low fields and an $\exp \left(B_{\|}\right)$dependence 


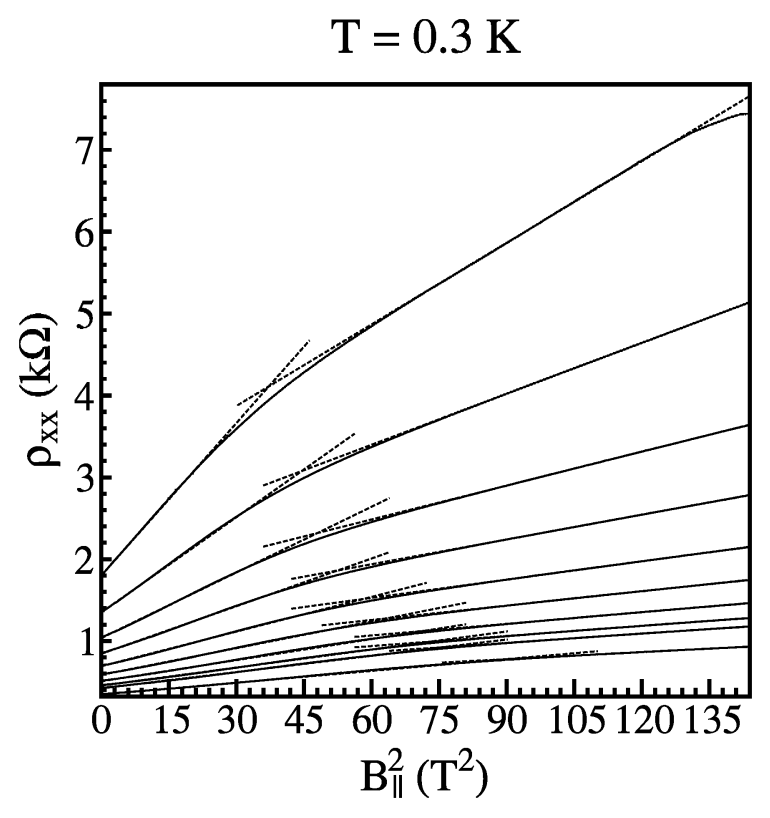

Fig. 2. $\rho_{x x}\left(B_{\|}^{2}\right)$ for various carrier densities. From top to bottom: $n_{\mathrm{S}}=1.379,1.481,1.591,1.688,1.780,1.884,1.967,2.036,2.076$ and $2.226 \times 10^{10} \mathrm{~cm}^{-2}$, respectively. Two linear fits for various $n_{\mathrm{s}}$ are shown in dotted lines.

at high fields. They showed that the onset of the $\exp \left(B_{\|}\right)$dependence corresponds to full spin polarisation in the $2 \mathrm{D}$ system $[19,20]$. These results $[19,20]$ justify the validity of our empirical identification in which the crossover of $B_{\|}^{2}$ dependence corresponds to full spin polarisation of our dilute GaAs electron system. As shown later, from $B_{\text {cross }}\left(n_{\mathrm{s}}\right)$ we can estimate the $g$-factor in our system. Note that the magetoresistivity shows little temperature dependence between $T=0.3 \mathrm{~K}$ and $T=1 \mathrm{~K}$, suggesting that the $B_{\|}^{2}$ dependence is a semi-classical effect. Also within our experimental accuracy, we did not observe a "metalinsulator transition (MIT)" in our device. The reason for this is probably due to the rather weak "MIT behaviour" in a 2D GaAs electron gas [21] and the limited measurement temperature range in our system.

Fig. 3 shows the crossing field $B_{\text {cross }}$ as a function of both carrier concentration $n_{\mathrm{s}}$ and the corresponding local Fermi energy $E$. Following the previous work $[15,16]$, we assume the slope of the $E-B_{\text {cross }}$ diagram is given by the Zeeman energy $E=\frac{1}{2} g \mu_{\mathrm{B}} B_{\|}$, where $\mu_{\mathrm{B}}$ is the Bohr magneton. In this case, a linear fit through the origin gives an estimated $g$-factor of 2.84 .

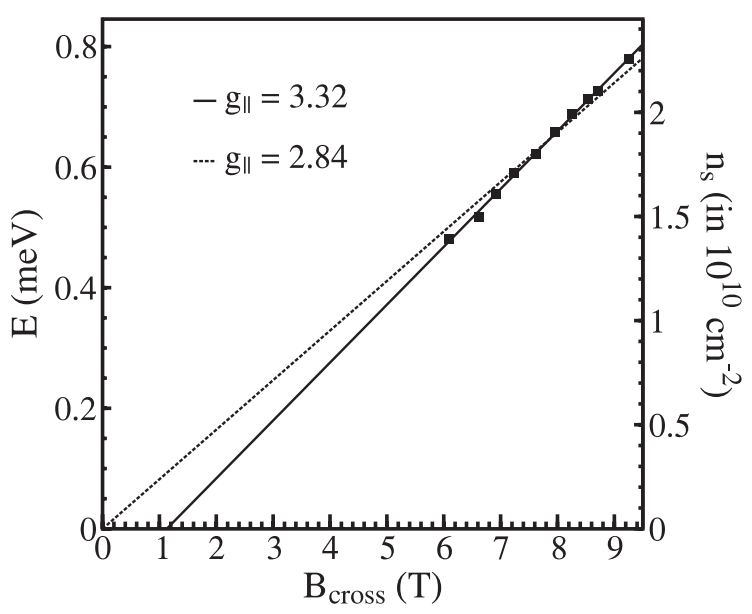

Fig. 3. Local Fermi energy $E$ and the corresponding 2D carrier density $n_{\mathrm{s}}$ at various measured crossing field $B_{\text {cross. }}$. The straight line fit through the origin is shown in the dotted line. The best linear fit is shown in the solid line.

As shown in Fig. 3, the best linear fit yields a value of the $g$-factor of 3.32. This fit gives a negative interception at $B=0$ which can be attributed to disorder broadening [16]. Note that both measured values are comparable to that measured in a clean 1D electron gas when there is a single 1D subband occupied [22]. Previously this enhancement of $g$-factor [22] is ascribed to electron-electron interactions at low carrier densities. We note that the dimensionaless parameter $r_{\mathrm{s}}$, the ratio of the Coulomb interaction energy to the kinetic (Fermi) energy reflects the strength of electronelectron interactions in the system. In our system, $r_{\mathrm{s}}$ is $\approx 4.7$ at the lowest carrier density and decreases to 3.7 at the highest $n_{\mathrm{s}}$. Therefore over the whole measurement range, $r_{\mathrm{s}}$ only decreases by an amount of $\approx 20 \%$. In this case, we believe that the strength of electronelectron interactions does not vary significantly over the whole measurement range, thus giving rise to an approximately constant $g$-factor determined from the straight line fit shown in Fig. 3.

Let us turn to our results on the mobility dependence on carrier density at different electron concentrations. If we take a vertical slice of the data shown in Fig. 1 , we can measure the resistivity (mobility) dependence on electron density for a fixed magnetic field. For the low $(B \leqslant 6 \mathrm{~T})$ and high $(B \geqslant 10 \mathrm{~T})$ magnetic field regimes, from the relation $\mu \propto n_{\mathrm{e}}^{\alpha}$, we can measure the exponent. For the intermediate magnetic field 


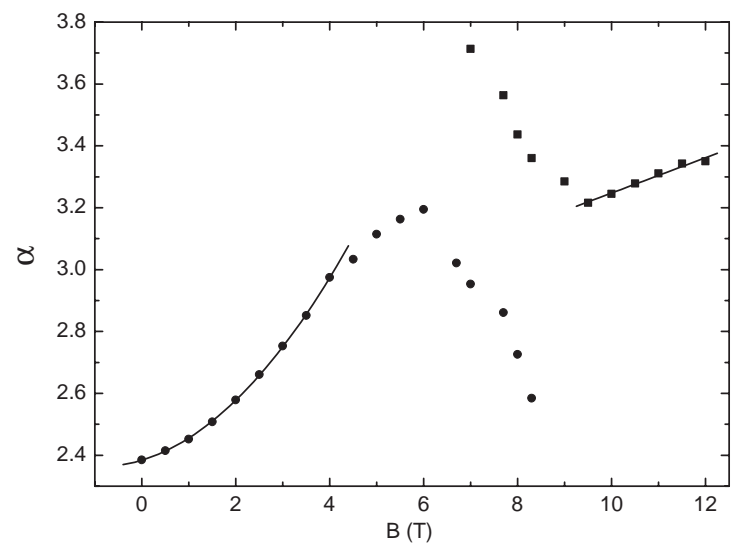

Fig. 4. The exponent $\alpha$ determined from the mobility dependence on carrier density. The circles correspond to the low-field regime and the squares correspond to the high-field regime. In the intermediate regime, there are two exponents as described in the text.

regimes $(6.7 \mathrm{~T} \leqslant B \leqslant 8.3 \mathrm{~T})$, the situation is somewhat complicate and is described as follows. There are two exponents in this intermediate regime. One is determined from the high $n$ side (labelled as circles) and the other is obtained from the low $n$ side (labelled as squares). In our system, at zero magnetic field, the exponent $\alpha$ is consistent with strong localisation at the $\mathrm{GaAs} / \mathrm{AlGaAs}$ interface [23]. With increasing in-plane magnetic field, $\alpha$ exhibits a $B^{2}$ dependence as shown in Fig. 4. In the intermediate regime, it is perhaps better to consider the averaged $\alpha \approx 3$. At high magnetic fields, $\alpha$ shows a linear $B$ dependence. In our system, the dilute $2 \mathrm{DEG}$ experiences larger scattering. Therefore at zero magnetic field $\alpha$ is larger than that $(\approx 1.5)$ of a typical 2DEG. Increasing the in-plane magnetic field increases the scattering in the 2DEG. This evidenced by the increase in $\rho_{x x}$ with increasing $B_{\|}$. Thus the exponent $\alpha$ increases with increasing $B_{\|}$.

In conclusion, we have measured a dilute gated 2D GaAs electron gas. Our experimental results obtained in a much weaker interacting GaAs electron system show that the magnetoresistance exhibits a much weaker $B_{\|}^{2}$ dependence compared with those in a GaAs hole gas and in a Si electron system. Using an empirical method, we estimate the Landé $g$-factor to be 3.32 in this dilute GaAs 2DEG. This enhanced $g$-factor is ascribed to electron-electron interactions and the fact that over the whole measurement range $r_{\mathrm{s}}$ does not vary significantly. We have also measured the exponent $\alpha$ in the relation $\mu \propto n^{\alpha}$ in our system.
It is found that $\alpha$ increases with increasing in-plane magnetic field. We ascribe this effect to the combination of increasing scattering and spin polarisation with increasing parallel magnetic field.

This work was funded by the NSC, Taiwan. The work at Cambridge was supported by the Engineering and Physical Sciences Research Council (EPSRC), UK. C.T.L. acknowledges support from the Royal Society (UK) and the Research Council, National Taiwan University, Taiwan. M.Y.S. acknowledges financial support from the ARC, Australia.

\section{References}

[1] A.B. Fowler, F.F. Fang, W.E. Howard, P.J. Stiles, Phys. Rev. Lett. 16 (1966) 901.

[2] F.F. Fang, P.J. Stiles, Phys. Rev. 174 (1968) 823.

[3] J.F. Janak, Phys. Rev. 178 (1969) 1416.

[4] K. Suzuki, Y. Kawamoto, J. Phys. Sco. Japan 35 (1973) 1456.

[5] T. Ando, Y. Uemura, J. Phys. Soc. Japan 37 (1974) 1044.

[6] C.S. Ting, T.K. Lee, J.J. Quinn, Phys. Rev. Lett. 34 (1975) 870.

[7] T. Ando, Phys. Rev. B 13 (1976) 3468.

[8] R. Dingle, H.L. Stormer, A.C. Gossard, W. Wiegmann, Appl. Phys. Lett. 33 (1978) 665.

[9] Th. Englert, D.C. Tsui, A.C. Gossard, Ch. Uihlein, Surf. Sci. 113 (1982) 295.

[10] R.J. Nicholas, R.J. Haug, K. von Klitzing, G. Weimann, Phys. Rev. B 37 (1988) 1294.

[11] A. Usher, R.J. Nicholas, J.J. Harris, C.T. Foxon, Phys. Rev. B 41 (1990) 1129.

[12] D. Simonian, S.V. Kravchenko, M.P. Sarachik, V.M. Pudalov, Phys. Rev. Lett. 79 (1997) 2304.

[13] M.Y. Simmons, A.R. Hamilton, M. Pepper, E.H. Linfield, P.D. Rose, D.A. Ritchie, A.K. Savchenko, T.G. Griffiths, Phys. Rev. Lett. 80 (1998) 1292.

[14] T. Okamoto, K. Hosoya, S. Kawaji, A. Yagi, Phys. Rev. Lett. 82 (1999) 3875.

[15] J. Yoon, C.C. Li, D. Shahar, D.C. Tsui, M. Shayegan, Phys. Rev. Lett. 84 (2000) 4421.

[16] V.M. Pudalov, G. Brunthaler, A. Prinz, G. Bauer, Phys. Rev. Lett. 88 (2002) 076401.

[17] S. Das Sarma, E.H. Hwang, Phys. Rev. Lett. 83 (1999) 164.

[18] V.T. Dolgopolov, A. Gold, JETP Lett. 71 (2000) 27.

[19] E. Tutuc, E.P. De Poortere, S.J. Papadakis, M. Shayegan, Physica E 2-4 (2002) 748.

[20] E. Tutuc, E.P. De Poortere, S.J. Papadakis, M. Shayegan, Phys. Rev. Lett. 86 (2001) 2858.

[21] Y. Hanein, D. Shahar, J. Yoon, C.C. Li, D.C. Tsui, H. Shtrikman, Phys. Rev. B 58 (1998) 13338.

[22] K.J. Thomas, J.T. Nicholls, M.Y. Simmons, M. Pepper, D.R. Mace, D.A. Ritchie, Phys. Rev. Lett. 77 (1996) 135.

[23] C.T. Foxon, J.J. Harris, R.G. Wheeler, D.E. Lacklison, J. Vac. Sci. Technol. B 4 (1986) 511. 